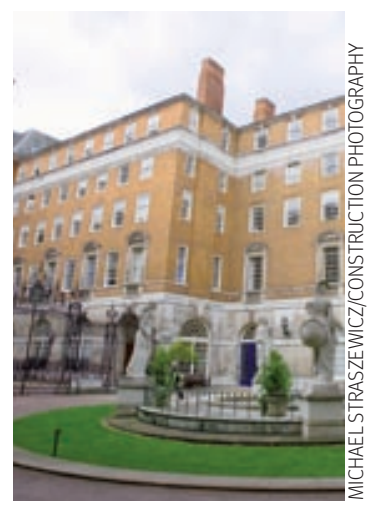

Roger Jones Wolfson professor of general practice Department of General Practice and Primary Care, KCL School of Medicine, London SE11 6SP roger.jones@kcl.ac.uk

Competing interests: None declared Provenance and peer review: Commissioned; not externally peer reviewed.

BMJ 2007:335:53 doi:10.1136/bmi.39266.662928.BE

\title{
The future of the medical profession
}

\author{
Depends on professional unity and respectful dialogue between the government \\ and the profession
}

This year marks the 175th anniversary of the BMA. The association has been centrally involved in the evolution and stewardship of the medical profession and has made contributions to national and international health that go far beyond its role as a representative and negotiating body. It has had an important role in debates on abortion, euthanasia, global conflict and the proliferation of nuclear weapons, AIDS, genetics in medicine, and human rights. It must also take a good deal of the credit for the present ban on smoking ${ }^{1}$ and, of course, for this journal.

The BMA took a highly conservative and aggressive stance against Bevin's plans for the National Health Service (NHS) in the 1940s, but 50 years later found itself almost in step with the health departments after the abolition of the internal market. However, this sense of common purpose is now much more difficult to discern.

The BMA has been faced with many difficulties in engaging with the complexities of 21 st century medicine and a highly politicised health service. The Shipman, Bristol, and Alder Hey enquiries, ${ }^{2-4}$ and a litany of errors, shook the foundations of public trust and professional confidence. Against a background of escalating healthcare costs, rapidly changing workforce and population demography, and the impact of the European Union, there have been recent difficulties with computerisation of the NHS, the postgraduate training system, doctors' contracts, and revalidation. The role of doctors' careers in medicine, and the future of a publicly funded health service have all been questioned.

It is ironic that some countries where the commercialisation of health care has developed unchecked, and others wishing to develop cost effective equitable health systems, have looked to the NHS as a role model. The NHS has, historically, provided universal coverage, ready access, and high quality care for an enviably low proportion of gross domestic product, although this is beginning to change. The reason for this change seems to be the political manipulation of the NHS by a health department that either does not understand or has forgotten what has made the NHS a success. It has disastrously underestimated the extent and importance of hard work, commitment, professionalism, and pride in the service among its workforce. This has resulted in a progressive deconstruction of general practice, the transformation of hospital contracts into a shift system, the introduction of bizarre organisational arrangements in the name of patient choice, and widespread demotivation and demoralisation. ${ }^{56}$

Has the medical profession, in its retreat from out of hours responsibility and personal continuity of care, its support of market values, and its acceptance of central control finally sold out? Or, is it that the NHS reforms have somehow eroded the conditions under which professionalism and altruism flourish? Altruism is, after all, sustained by appreciation on both sides, ${ }^{7}$ just as professional respect and esteem are earned by the provision of compassionate care.

To say that the medical profession must unite to reaffirm its core values sounds like a statement of the obvious, but it is probably right. The profession certainly can't afford division. Ever since the NHS was formed, there has been an inevitable separation between primary care (general practice and community medicine) and secondary care (hospital medicine), which has often caused tension between professionals. Current funding arrangements-including practice based commissioning ${ }^{8}$ and payment by results ${ }^{9}-$ could aggravate these differences. Similarly, friction between doctors and health service managers needs to be tackled by collaborative working, including greater participation of senior clinicians in the management of health services.

Medical education and the nurturing of professional values have an important part to play, particularly in moving towards a "new compact" between patients and doctors, put forward by Ham and Alberti, which spells out the rights and responsibilities of the government, the profession, and the public. ${ }^{10}$ Serious consideration should be given to proposals made recently to take the NHS out of party politics by creating new governance arrangements. ${ }^{11}$ All of this must be firmly underpinned by authoritative and consistent dialogue between the profession and the media.

These suggestions do not represent an agenda for a return to some lost, golden age of medicine, but rather an opportunity to reassert its enduring roles and values, which have been obscured by political and organisational turbulence. After the Cabinet Office's capability review of the Department of Health, ${ }^{12}$ there are signs that a new political administration may consider better ways of working with medical leaders and the NHS. The importance of doing so cannot be underestimated. 


\section{Diarrhoea associated with antibiotic use}

\section{Evidence supports the use of probiotics, but effectiveness depends on the strain}

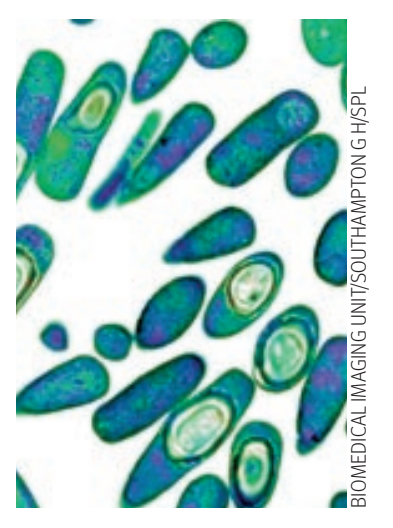

RESEARCH, p 80

Lynne V McFarland affiliate associate professor Department of Health Services Research and Development, Puget Sound Veteran Administration Healthcare System, 1100 Olive Way \#1400, Seattle, WA 98101, USA Ivmcfarl@u.washington.edu

Competing interests: LVM has been reimbursed by Laboratories Biocodex and Klaire Labs for attending several conferences and for speaker fees. The viewpoints in this article are solely those of the author and do not represent the viewpoints of the Veterans Administration.

Provenance and peer review:

Commissioned; not externally peer reviewed.

BMJ 2007:335:54-5 doi: 10.1136/bmj.39255.829120.47
Diarrhoea is a common side effect of antibiotics; it may prolong hospital stay, increase the risk of other infections, develop into more serious forms of disease (colitis, toxic megacolon), and lead to premature discontinuation of the needed antibiotic. Diarrhoea associated with Clostridium difficile is a leading cause of iatrogenic outbreaks of diarrhoea, and considerably increases mortality and healthcare costs for inpatients. ${ }^{1-4}$ Antibiotic associated diarrhoea may develop in 5-30\% of patients, with the rates increasing as the antibiotic spectrum gets broader. $^{4}$

Diarrhoea associated with antibiotic use may result from the disruption of the barrier of normally protective colonic microflora that are inadvertent targets of the inciting antibiotic. In 20-30\% of these cases, an opportunistic pathogen, Clostridium difficile, takes advantage of this opening, colonises the intestine, and produces toxins, resulting in diarrhoea or colitis. A strategy to re-establish this microbial barrier is through the use of probiotics. ${ }^{5}$ In this week's BMJHickson and colleagues report a randomised, placebo-controlled trial of the effects of a mixture of three different strains of probiotics on prevention of diarrhoea associated with antibiotic use. ${ }^{6}$

Probiotics are living, beneficial bacteria or yeasts that are taken orally to help restore the microbial balance in the intestinal tract. ${ }^{7}$ A multitude of probiotic products are available in the global marketplace, but only some are backed by evidence based clinical trials. ${ }^{8-12}$ The diversity of probiotic products has caused confusion among clinicians and patients as to which are effective and which are not.

The trial by Hickson and colleagues randomised 135 older hospitalised patients receiving a new course of antibiotics to either a probiotic yoghurt drink (containing Lactobacillus casei DN 114 001, L bulgaricus, and Streptococcus thermophilus) or a placebo milkshake for the duration of the antibiotic plus one week. Patients were followed for an additional four weeks for the development of antibiotic associated diarrhoea or $C$ difficile diarrhoea. Significantly fewer patients given the probiotic drink developed diarrhoea than did those given the placebo milkshake (odds ratio $0.25,95 \%$ confidence interval 0.07 to 0.85 ). Although the rates of $C$ difficile diseases were low, the probiotic drink also seemed to prevent this outcome $(0 \%$ v 17\% with placebo; adjusted rate ratio $17 \%$, $7 \%$ to $27 \%$ ). No adverse effects were reported.

The trial has several strengths: the outcomes (diarrhoea associated with antibiotic use and $C$ difficile diarrhoea) were defined, the doses (number of bacteria per day) of probiotics were provided, compliance was assessed, and treatments were blinded to patients and assessors.

The trial is limited by its low generalisability due to the small proportion $(8 \%)$ of people who were enrolled from the target pool of hospitalised patients receiving antibiotics. For a preventive treatment to be practical, it needs to be given widely to the population at risk.
Another limitation is the lack of proper probiotic designations. Closely related bacterial strains have been shown to have differing abilities to act as efficacious probiotics, ${ }^{13-15}$ so the need to correctly identify the strain (not just the genus and species) of probiotic under investigation is paramount. The authors identified only one strain (L casei DN 114 001), and they (inappropriately) cited its brand name as part of its nomenclature. A description of the time of onset would have been helpful (while patient was taking antibiotics or delayed onset after antibiotics were stopped) so that clinicians know when they should expect diarrhoea to develop. The authors also say their analysis was intention to treat, but patients lost to follow-up were not included; although the loss to follow-up was moderate (22 patients; $16 \%$ ) and the numbers were similar in the two groups.

Despite these limitations, this study supports the findings from other randomised, double blinded, controlled trials that various probiotic preparations are effective for preventing diarrhoea associated with antibiotic use..$^{8-12}$

So how does this growing body of evidence translate into clinical practice? The study by Hickson and colleagues adds to the findings from recent meta-analyses that probiotics are effective for preventing diarrhoea associated with antibiotics. ${ }^{11}{ }^{12}$ Some European hospitals routinely recommend probiotics as an adjunct to high risk antibiotics. Probiotics may be especially useful for patients with chronic infections (such as sinusitis or diabetic foot ulcers) that require repeated courses of antibiotics. Barriers to this practice include choosing an effective probiotic strain, the additional cost of the probiotic, and risks associated with use in immunocompromised patients. Probiotics can easily be given along with antibiotics as an adjunctive preventive treatment and seem to be well tolerated by both paediatric and elderly populations. ${ }^{12} 1416$ A word of caution: clinicians need to consider which probiotic strain is supported by evidence from clinical trials, as not all probiotics have equal effectiveness. The evidence that probiotics are effective as a preventive measure for Clostridium difficile requires further trials, and more strains need to be tested for their probiotic potential.

1 Shek FW, Stacey BS, Rendell J, Hellier MD, Hanson PJ. The rise of Clostridium difficile: the effect of length of stay, patient age and antibiotic use. J Hosp Infect 2000;45:235-7.

2 Lawrence SJ, Puzniak LA, Shadel BN, Gillespie KN, Kollef MH, Mundy LM. Clostridium difficile in the intensive care unit: epidemiology, costs, and colonization pressure. Infect Control Hosp Epidemiol 2007;28:12330.

3 Pépin J, Valiquette L, Cossette B. Mortality attributable to nosocomial Clostridium difficile-associated disease during an epidemic caused by a hypervirulent strain in Quebec. CMA/ 2005;173:1037-42.

4 McFarland LV, Beneda HW, Clarridge JE, Raugi GJ. Implications of the changing face of Clostridium difficile disease for health care practitioners. Am J Infect Control 2007;35:237-53.

5 McFarland LV. Normal flora: diversity and functions. Microb Ecol Health Dis 2000;12:193-207.

6 Hickson M, D'Souza AL, Muthu N, Rogers TR, Want S, Rajkumar C, et al. Use of probiotic Lactobacillus preparation to prevent diarrhoea 
associated with antibiotics: randomised double blind placebo controlled trial. BMJ 2007 doi: 10.1136/bmj.39231.599815.55

7 Elmer GW, McFarland LV, McFarland M. Introduction. In: The power of probiotics: improving your health with beneficial microbes. Binghamton, NY: Haworth Press, 2007:1-24.

8 Huebner ES, Surawicz CM. Probiotics in the prevention and treatment of gastrointestinal infections. Gastroenterol Clin North Am 2006;35:355-65.

9 Johnston B, Supina A, Ospina M, Vohra S. Probiotics for the prevention of pediatric antibiotic-associated diarrhea. Cochrane Database Syst Rev 2007;(2):CD004827.www.cochrane.org/reviews/en/ab004827.htm

10 Katz IA. Probiotics for the prevention of antibiotic-associated diarrhea and Clostridium difficile diarrhea. J Clin Gastroenterol 2006;40:249-55.

11 McFarland LV. Meta-analysis of probiotics for the prevention of antibiotic associated diarrhea and the treatment of Clostridium difficile disease. Am J Gastroenterol 2006;101:812-22.

12 Szajewska H, Ruszczy ski M, Radzikowski A. Probiotics in the prevention of antibiotic-associated diarrhea in children: a meta- analysis of randomized controlled trials. I Pediatr 2006:149:367-72.

13 Mountzouris KC, Tsirtsikos P, Kalamara E, Nitsch S, Schatzmayr $\mathrm{G}$, Fegeros K. Evaluation of the efficacy of a probiotic containing Lactobacillus, Bifidobacterium, Enterococcus, and Pediococcus strains in promoting broiler performance and modulating cecal microflora composition and metabolic activities. Poult Sci 2007;86:309-17.

14 Hutt P, Shchepetova J, Loivukene K, Kullisaar T, Mikelsaar M. Antagonistic activity of probiotic lactobacilli and bidifobacteria against entero- and uropathogens. J Appl Microbiol 2006;100:1324-32.

15 Timmerman HM, Niers LE, Ridwan BU, Koning CJ, Mulder L, Akkermans $\mathrm{LM}$, et al. Design of a multispecies probiotic mixture to prevent infectious complications in critically ill patients. Clin Nutr 2007 June 4; doi: 10.1016/j.clnu.2007.04.008

16 McFarland LV, Elmer GW, McFarland M. Meta-analysis of probiotics for the prevention and treatment of acute pediatric diarrhea. IntlJ Probiotics Prebiotics 2006;1:63-76.

\section{Surgery for venous leg ulcers}

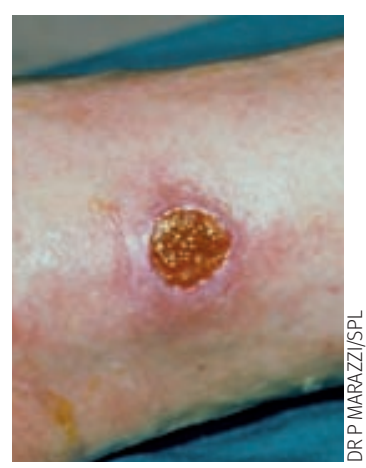

RESEARCH, p 83

Charles N McCollum professor of surgery

Education and Research Centre, University Hospital of South Manchester, Manchester M239LT cnmcc@manchester.ac.uk Competing interests: None declared. Provenance and peer review: Commissioned; not externally pee reviewed.

BMJ 2007;335:55-6 doi: 10.1136/bmj.39261.651655.47

\section{Can reduce recurrence, but will have little impact on prevalence}

Venous leg ulcers are painful, malodorous sores that impair quality of life and are difficult to treat. ${ }^{1}$ An estimated $5-8 \%$ of the world's population have venous disease, and $1 \%$ have venous ulcers at some time in their life. ${ }^{2}$ The cost to healthcare services is best known for the United Kingdom, where active ulcers affect $1.7 \%$ of the elderly population, at a cost to the NHS of around $£ 600 \mathrm{~m}$ (€890m; \$1200m) a year. ${ }^{34}$ Available evidence suggests costs are high throughout Europe, the United States, and Australia. These ulcers are caused by sustained high venous pressures due to venous disease, obesity, immobility associated with arthritis, or even old age itself.

Compression using four layer bandaging is the mainstay of treatment-it completely heals ulcers in a mean of 7-8 weeks when delivered by trained leg ulcer nurses in the community. ${ }^{56}$ The efficacy of four layer bandaging is not influenced by the underlying venous abnormality. ${ }^{7}$ Whether novel "biologically active" dressings can improve these healing rates remains uncertain, as does the role of venous surgery.

In this week's BMJ Gohel and colleagues report the long term results from the ESCHAR trial, which compared compression alone with compression plus superficial venous surgery in patients with open or recently healed leg ulcers and superficial venous incompetence. ${ }^{8}$ This trial was adequately powered and reported on ulcer healing, ulcer recurrence, and ulcer-free time over three or four years. Most previous trials either ignored the role of compression therapy or compared surgery with compression, which is inappropriate as both are effective treatments that should be complementary. ${ }^{568}$

ESCHAR clarifies the role of superficial venous surgery in people willing or able to have an operation. ${ }^{8}$ The trial found no significant difference between compression alone and compression plus surgery on ulcer healing at three years-but recurrence, which otherwise happens in a quarter of patients each year, was almost halved. This beneficial effect was most obvious in patients who have incompetence affecting only the superficial veins or those with "segmental" deep venous incompetence, in which reflux is found in limited segments of the deep veins without widespread valve failure. The authors' use of "isolated superficial" where the deep veins are normal, "segmental deep," and "total deep" incompetence is unfortunate as most "segmental deep" incompetence is reflux within the common femoral or popliteal vein emptying into the incompetent long or short saphenous vein; valve failure is largely confined to the superficial veins. ${ }^{9}$

The finding of reduced ulcer recurrence after superficial vein surgery in patients with "total deep" incompetence is surprising as valve failure causes widespread deep venous reflux in such patients. However, early results from the ESCHAR study showed that ablating incompetent superficial veins improves deep venous function. ${ }^{10}$ This does not mean that all patients with combined superficial and deep incompetence would benefit from superficial surgery. ${ }^{9}$ Preoperative assessment in ESHCAR was by duplex imaging, which describes the anatomy of venous disease but not the function. Ambulatory venous pressures would be a more reliable measure of venous function, with a narrow tourniquet obstructing the superficial veins. ${ }^{9}$ Many surgeons do not use ambulatory venous pressure measurement because it requires cannulation of the foot, even though it is the only direct measure of venous pressures.

The encouraging results of surgery on ulcer recurrence rates in the ESCHAR trial will sadly have little influence on overall ulcer prevalence. Patients were recruited from specialist, hospital based leg ulcer clinics, whereas many elderly people in the community refuse to attend hospitals for either venous investigations or surgery. ${ }^{6} 11$ Even among the hospital clinic patients screened for ESCHAR, over a third refused to be randomised and a further $20 \%$ refused surgery despite consenting to the study. ${ }^{8}$ In the community, our experience is that less than half the patients would attend for investigation, and perhaps a third would consider surgery, simple pinch skin grafting being an exception as it can be done in the community by specialist nurses, under local anaesthetic, and speeds the healing of large ulcers. ${ }^{12}$

What does this mean for general practitioners looking after people with leg ulcers? Firstly, people should be referred to a specialist leg ulcer service for investigation of arterial disease before four layer bandaging, if appropriate. 
These services should include a local vascular surgeon to manage arterial disease and arrange venous investigations in people being considered for surgery or, in the future, microfoam sclerotherapy or endovenous laser treatment. Referral to a vascular surgeon does not need to be delayed until the ulcer has healed, and nor should a history of deep vein thrombosis be a deterrent since it is found in only $10 \%$ of people with leg ulcers, and in a third of those with deep venous incompetence. ${ }^{6}$ Superficial venous incompetence, the usual cause of varicose veins, can be detected in most patients with venous ulcers and is a potentially correctible cause of venous hypertension. ${ }^{8}$ Neither ESCHAR nor a previous study found that superficial venous surgery accelerates healing. ${ }^{813}$ However, there is no need to delay venous surgery where appropriate for uninfected leg ulcers.

Future research should focus on identifying patients at risk of ulceration in order to prevent rather than treat. Superficial venous surgery and compression will almost certainly both have a role in ulcer prophylaxis.

1 Iglesias CP, Birks Y, Nelson EA, Scanlon E, Cullum NA. Quality of life of people with venous leg ulcers: A comparison of the discriminative and responsive characteristics of two generic and disease specific instruments. Qual Life Res 2005;14:1705-18.

2 Ruckley CV. Socioeconomic impact of chronic and venous insufficiency and leg ulcers. Angiology 1997; 48:67-9.

3 Douglas WS, Simpson NB. Guidelines for the management of chronic venous leg ulceration. Report of a multidisciplinary workshop. British
Association of Dermatologists and the Research Unit of the Royal College of Physicians. BrJ Dermatol 1995;132:446-52.

4 Margolis DJ, Bilker W, Santanna J, Baumgarten M. Venous leg ulcer: incidence and prevalence in the elderly. J Am Acad Dermato 2002;46:381-6

5 Blair SD, Wright DD, Backhouse CM, Riddle E, McCollum CN. Sustained compression and healing of chronic venous ulcers. BMJ 1988;297:1159-61.

6 Moffatt CJ, Franks PJ, Oldroyd M, Bosanquet N, Brown P, Greenhalgh RM, et al. Community clinics for leg ulcers and impact on healing. BMJ 1992;305:1389-92.

7 Guest M, Smith JJ, Sira MS, Madden P, Greenhalgh RM, Davies $\mathrm{AH}$. Venous ulcer healing by four-layer compression bandaging is not influenced by the pattern of venous incompetence. Br / Surg 1999;86;1437-40.

8 Gohel MS, Barwell JR, Taylor M, Chant T, Foy C, Earnshaw JJ, et al. Long term results of compression therapy alone versus compression plus surgery in chronic venous ulceration (ESCHAR): randomised controlled trial. BM/ 2007doi:10.1136/bmj.39216.542442.BE.

9 Dix FP, Picton A, McCollum CN. Effect of superficial venous surgery on venous function in chronic venous insufficiency. Ann Vasc Surg 2005;19:678-85.

10 Gohel MS, Barwell JR, Earnshaw JJ, Heather BP, Mitchell DC, Whyman $M R$, et al. Randomized clinical trial of compression plus surgery versus compression alone in chronic venous ulceration (ESCHAR study) haemodynamic and anatomical changes. Br/Surg 2005;92:291-7.

11 Freak L, Simon D, Kinsella A, Walsh J, Lane C, Groarke L, McCollum CN. Community leg ulcer clinics: comparative study of two health authorities. BM/1996;312:164-5.

12 Poskitt KR, James AH, Lloyd-Davies ERV, Walton J, McCollum CN. Pinch skin grafting or porcine dermis in venous ulcers: A randomized clinical trial. BMJ 1987;294:674-7.

13 Guest M, Smith JJ, Tripuraneni G, Howard A, Madden P, Greenhalgh $\mathrm{RM}$, et al. Randomized clinical trial of varicose vein surgery with compression versus compression alone for the treatment of venous ulceration. Phlebology 2003;18:130-6.

\section{Treating rheumatoid arthritis}

\section{Antitumour necrosis factor can produce remission if started early}

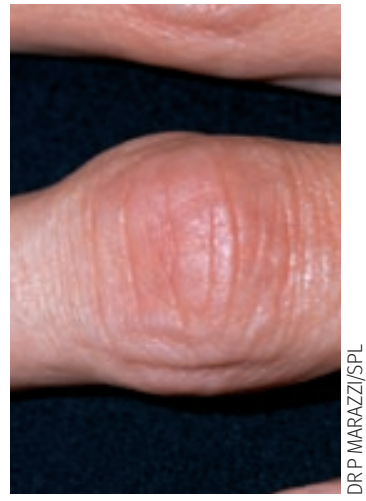

Paul Emery professor and head of academic unit of musculoskeletal disease

Leeds University, Leeds LS7 4S

Tore K Kvien professor and head of rheumatology department

Department of Rheumatology,

Diakonhjemmet Hospital and Faculty of

Medicine, University of Oslo, Norway

P.Emery@leeds.ac.uk

Competing interests: PE and

TKK have provided expert advice

and undertaken clinical trials for

producers of biological agents.

Provenance and peer review:

Commissioned; not externally pee reviewed.

\section{BMJ 2007;335:56-7}

doi: 10.1136/bmj.39265.679375.80
Rheumatoid arthritis is a chronic inflammatory disease affecting the synovial joints. It is characterised by persistent inflammation and destruction of bone and joints. It affects physical functioning and other dimensions of quality of life. Symptoms can be treated with analgesics and non-steroidal anti-inflammatory drugs, which block cyclo-oxygenase. The underlying disease process can be affected by drugs that block or reduce the concentration of cytokines, which are known as disease modifying antirheumatic drugs. These drugs are either conventional small molecules or biological molecules.

The outcome of treatment for patients with rheumatoid arthritis has improved considerably during the past 20 years. ${ }^{1}$ People have speculated about the potential to reverse the disease for some time. Treatments that aim to induce remission have been called for since the start of the 1990s. ${ }^{2}$ Several approaches have been investigated, including step-up (adding one drug to another) and step-down (starting with a combination of drugs and withdrawing individual ones) regimens ${ }^{3}$ and giving three disease modifying antirheumatic drugs at the same time. ${ }^{4}$ All approaches have reduced inflammatory activity, delayed radiographic progression, and improved function and quality of life. However, only a few patients have achieved remission. ${ }^{5}$

The benefits of cytokine blockers-for example, drugs that antagonise tumour necrosis factor (TNF)-have exceeded initial predictions. Blocking TNF was predicted to reduce symptoms, but to have little structural effect on bone and cartilage, as evidence from animal models indicated that interleukin-1 was a more important bone cytokine than TNF. Clinical studies have shown, however, that TNF blocking agents have long lasting benefit, particularly when combined with methotrexate, and have a major effect on bone damage. ${ }^{6}$ There is thus little doubt that the current most effective treatment for patients with rheumatoid arthritis is a combination of methotrexate and anti-TNF. But questions remain, such as whether all patients need this treatment to achieve an adequate response and whether it can be cost effective.

The effects of TNF on remission have been assessed in two studies. ${ }^{78}$ The first found that 12 months of antiTNF given early on in the disease process could produce remission, which was sustained one year after ceasing treatment. ${ }^{7}$ The second found that six months of TNF blockade showed only short term benefit. ${ }^{8}$

Against this background, a single blinded pragmatic study with four treatment arms was started in the Netherlands, and the longer term data from this study have now been published. ${ }^{9}$ The first two arms tested the efficacy of conventional disease modifying antirheumatic drugs, with either a switching or step-up protocol, while the third and fourth arms compared anti-TNF plus methotrexate with combination treatment plus corticosteroids.

A crucial feature of the study was that participants were all treated according to prespecified targets (treatment-to-target strategy)-initial drug regimens 
were adjusted if participants failed to reach low disease activity (disease activity score $\leq 2.4$ ). By definition, all patients should have achieved the same end point, as they would have been switched to a biological treatment if they did not respond. The design allowed the investigators to analyse how frequently the strategy could succeed and to examine subsets of patients for the benefits of strategies such as switching or stepping down drugs.

What were the main findings of this study? Firstly, by using an aggressive treatment-to-target strategy, remission can be achieved in about $40 \%$ of patients. Secondly, the trial arms that included anti-TNF reduced inflammation more rapidly, reduced long term disease activity more effectively, and had bigger structural benefits than those that did not. Thirdly, induction with anti-TNF allowed more patients to come off the biological treatment and remain in remission. Fourthly, the escalating monotherapy arms (which measured the likelihood of a good response to individual agents) showed that while around $30 \%$ of patients started on methotrexate responded well, subsequent responses to further conventional disease modifying antirheumatic drugs were rare. Thus, most patients who fail methotrexate monotherapy will benefit from a direct switch to anti-TNF plus methotrexate, rather than trying other conventional antirheumatic drug regimens.

These long term data confirm the success of the treatment-to-target strategy, which has improved outcome in other pragmatic trials with conventional disease modifying antirheumatic drugs. ${ }^{10}$ Furthermore, anti-TNF plus methotrexate was the most effective and least toxic treatment.

Another interesting message to come out of the study relates to prognosis. Patients entered into this study had established early rheumatoid arthritis and a high proportion had structural damage, indicating disease with a relatively poor prognosis. It is interesting that patients who declined entry into the study, and mostly had milder disease at baseline (and should therefore have had a better outcome), ended up doing much worse than the included patients (F Breedveld, personal communication, 2007). One lesson is that if patients with a poor prognosis could be identified early, then they would be the ones most likely to benefit from more aggressive approaches.

Data suggest that patients with a poor prognosis can be identified by a combination of features including a high acute phase response, autoantibodies to rheumatoid factor or cyclic citrullinated peptides (or both), and evidence of radiological erosions at baseline. By using more sensitive imaging techniques, it might be possible to identify more people at high risk of a severe disease course. ${ }^{11}$

Are we now in a position to recommend that patients with a poor prognosis should receive anti-TNF as first line treatment? If the cost of the drugs was minimal, the only relevant comparator would be combination regimens with corticosteroids and methotrexate as the anchoring drugs. Cost effectiveness analyses are under way and will influence how widespread such an approach is likely to be.

The success of the treatment-to-target strategy supports its use in clinical practice whenever possible. The data also suggest that patients with rheumatoid arthritis can achieve remission, but for them to cease treatment and remain in remission they must be treated early on in the disease process. ${ }^{712}$ However, sufficient uncertainty exists to warrant further double blinded trials and analyses of their costs.

\section{Treating painful diabetic polyneuropathy}

\section{As consensus is lacking, protocols need to be devised and implemented locally}

\section{RESEARCH, p 87}

Edward B Jude consultant physician and honorary senior lecturer Tameside General Hospital, Ashton-under-Lyne,

Lancashire OL6 9RW

Nicolaas Schaper professor

Division of Endocrinology,

Department of Internal Medicine,

University Hospital Maastricht, PO

Box 5800, 6202 AZ

Maastricht, Netherlands

Edward.Jude@tgh.nhs.uk

Competing interests: E) has received

funding for research, speaker fees

and support for education from

Pfizer and speaker fees from

Boehringer Ingelheim.

Provenance and peer review:

Commissioned; not externally peer reviewed.

BMJ 2007;335:57-8

doi: 10.1136/bmj.39261.687650.47
The incidence of diabetes is expected to double over the next two decades, which will result in more people with complications of diabetes. ${ }^{1}$ Diabetic polyneuropathy is one of the most common, with a prevalence of around 30-50\%. It can have a major impact on patients' quality of life, and treatment is usually needed for many years. ${ }^{2} 3$

Community based studies report the prevalence of painful diabetic polyneuropathy as around $16-26 \%,{ }^{4}$ and in one study $80 \%$ of the patients had moderate or severe pain. ${ }^{5}$ Quality of life is reduced in patients with painful diabetic polyneuropathy, with restriction in daily and social activities, and the condition is associated with depression, sleep disturbances, and anxiety. ${ }^{367}$ In this week's $B M J$ Wong and colleagues report a systematic review of the effects of drug treatment in painful diabetic polyneuropathy. ${ }^{8}$

Many types of drugs have been studied for relief of pain in diabetic polyneuropathy, ${ }^{9}$ as little evidence exists that classic analgesics such as paracetamol or non-steroidal anti-inflammatory drugs are effective. Surveys show that there is substantial scope for improvement in clinical care. ${ }^{6}{ }^{10}$ In one UK population based study almost $40 \%$ of people with painful neuropathy reported that they had never received any treatment, almost a third had been prescribed drugs with no known efficacy in neuropathic pain, and only a minority had been treated with tricyclic antidepressants or anticonvulsants. ${ }^{4}$

Wong and colleagues' review identified the randomised placebo controlled trials of topically applied and orally administered drugs. ${ }^{8}$ Clinical success was defined as a $50 \%$ reduction in pain; withdrawal due to adverse events was a secondary outcome. The review found that tricyclic antidepressants were most effective in reducing pain by $50 \%$ (odds ratio 31.73 ; $95 \%$ confidence interval 3.68 to 189.89 ), followed by traditional anticonvulsants (lamotrigine, sodium valproate, carbamazepine; odds ratio $7.59 ; 2.16$ to 26.58), and the newer generation anticonvulsants 
(gabapentin, oxacarbazepine, pregabalin; odds ratio $3.25 ; 2.27$ to 4.66$)$. The newer generation anticonvulsants were most likely to cause withdrawals due to adverse events, followed by tricyclic antidepressants, and the traditional anticonvulsants (odds ratios 2.98, 2.32, 1.51 respectively).

This review can help clinicians make evidence based choices in the management of painful diabetic polyneuropathy, and it also highlights some of the problems in the treatment regimens often prescribed for this condition. The efficacy of pharmacological treatment is limited: with of one of the most efficacious drugs in the meta-analysis, amitriptyline, three patients need to be treated to achieve a $50 \%$ reduction in pain score in one patient; in the other two patients the drug will have no effect or a limited effect. Adverse effects were not infrequent, and in the analysis of the effects of tramadol the odds ratio for withdrawal due to adverse events was higher than the odds ratio for $50 \%$ pain relief. It is difficult to compare the results of different trials in the review since the duration of treatment was highly variable (between 2 to 16 weeks) and could have influenced the number of withdrawals. Unfortunately all the trials in the review were relatively short; many included only a few patients), and for some drugs the confidence intervals were large. Clearly, robust studies with sufficient size and duration, preferably of at least one year, are needed.

Treatment of painful diabetic polyneuropathy is a clinical challenge and requires a treatment plan that should include psychosocial factors, glucose control, and, if necessary, pharmacological treatment. Listening to the patient and explaining the cause of the pain can help to reduce anxiety. Patients' beliefs and perceptions of the pain and its cause, coping strategies, mood changes, disturbed sleep, and anxiety all need to be addressed.

Several studies have looked at the impact of analgesic treatment on quality of life, sleep patterns, anxiety, and depression and most of them have shown an improvement in quality of life which might be directly related to improvement in pain score. ${ }^{11}$ Therefore, treating anxiety or depression first might also reduce the need for analgesics. A few observational studies have indicated that large variations in blood glucose can exacerbate the pain, so glycaemic control should be optimised. ${ }^{12} 13$

If, despite these measures, the pain persists and is so severe that pharmacological treatment is indicated tricyclic antidepressants seem the best first step, as suggested by Wong and colleagues' review. But contraindications include cardiac conduction disturbances and glaucoma, and side effects are not infrequent. The dilemma in treating painful diabetic polyneuropathy is what should be done with the many people who do not respond sufficiently to tricyclic antidepressants or in whom tricyclic antidepressants are contraindicated. Wong and colleagues suggest that the next step should be to use one of the older generation anticonvulsants. However, this advice is based on clustering a group of anticonvulsants with very different modes of action; if they are analysed separately, the number of studies for each drug is relatively small and the evidence for efficacy does not seem convincing. Therefore, we and others suggest pregabalin, duloxetine, or gabapentin as second line agents, as these drugs do not seem to differ much in efficacy or in the frequency and severity of side effects. ${ }^{9}$ Only one or two of these drugs should be included in the local treatment protocol, so that clinicians can develop sufficient experience with them. If this treatment fails, combination therapy or tramadol (or another opioid) can be considered.

Studies that evaluate the efficacy of the combination of two or more drugs to relieve pain are few. Only one study combined gabapentin and morphine in people with neuropathic pain; it showed that the combination gave better pain relief, required lower doses of both drugs, and fewer side effects than if the drugs were used singly. ${ }^{14}$

Although our knowledge of the treatment of painful diabetic polyneuropathy is growing, there is still no consensus on the most effective protocol. Therefore doctors treating people with diabetes must develop their own local screening and treatment protocols. They should include a treatment algorithm, indications and contraindications for drug treatment, potential adverse effects to look out for, and a simple technique to monitor the effectiveness of treatment.

1 Wild S, Roglic G, Green A, Sicree R, King H. Global prevalence of diabetes: estimates for the year 2000 and projections for 2030 . Diabetes Care 2004;27:1047-53.

2 Jude E, Boulton AJM. End stage complications of diabetic neuropathy. Diabetes Rev 1999;7:395-410.

3 Vileikyte L, Rubin RR, Leventhal H. Psychological aspects of diabetic neuropathic foot complications: an overview. Misconceptions, assessment of patient beliefs and behaviour, illness perception at the individual level. Diabetes Metab Res Rev 2004;20:S13-8.

4 Daousi C, MacFarlane IA, Woodward A, Nurmikko TJ, Bundred PE, Benbow SJ. Chronic painful peripheral neuropathy in an urban community: a controlled comparison of people with and without diabetes. Diabet Med 2004;21:976-82.

5 Davies M, Brophy S, Williams R, Taylor A. The prevalence, severity, and impact of painful diabetic peripheral neuropathy in type 2 diabetes. Diabetes Care 2006;29:1518-22.

6 Gore M, Brandenburg NA, Hoffman DL, Tai KS, Stacey B. Burden of illness in painful diabetic peripheral neuropathy: the patients' perspectives. J Pain 2006;7:892-900.

7 Benbow SJ, Wallymahmed ME, MacFarlane IA. Diabetic peripheral neuropathy and quality of life. QJM 1998;91:733-7.

8 Wong M, Chung JWY, Wong TKS. Effects of treatments for symptoms of painful diabetic neuropathy: systematic review. BM/ 2007 doi:10.1136/bmj.39213.565972.AE.

9 Jensen TS, Backonja MM, Hernández Jiménez S, Tesfaye S, Valensi $P$, Ziegler D. New perspectives on the management of diabetic peripheral neuropathic pain. Diabetes Vasc Dis Res 2006;3:108-19.

10 Toelle T, Xu X, Sadosky AB. Painful diabetic neuropathy: a crosssectional survey of health state impairment and treatment patterns. J Diabetes Complicat 2006;20:26-33.

11 McCarberg B, Billington R. Consequences of neuropathic pain: quality of life issues and associated costs. Am J Manag Care 2006;12:S263-8.

12 Boulton AJM, Drury J, Clarke, Ward JD. Continous subcutaneous insulin infusion in the management of painful diabetic neuropathy. Diabetes Care 1982;5:386-90.

13 Oyibo S, Prasad YD, Jackson NJ, Jude EB, Boulton AJ. The relationship between blood glucose excursions and painful diabetic peripheral neuropathy. Diabet Med 2002;19:870-3.

14 Gilron I, Bailey JM, Tu D, Holden RR, Weaver DF, Houlden RL. Morphine, gabapentin, or their combination for neuropathic pain. N Engl J Med 2005;352:1324-34. 